Proceedings

\title{
Ultrahigh Carbon Nanotube Volume Fraction Effects on Micromechanical Quasi-Static \& Dynamic Properties of Poly(Urethane-Urea) Filled Nanocomposites ${ }^{+}$
}

\author{
Jeffrey L. Gair, Jr. 1,2,3, Dale L. Lidston ${ }^{3}$, Daniel P. Cole ${ }^{1}$, Robert H. Lambeth ${ }^{4}$, Alex J. Hsieh ${ }^{4}$, \\ Hugh A. Bruck ${ }^{2}$, Asha J. Hall ${ }^{1}$, Mark L. Bundy ${ }^{1}$ and Brian L. Wardle ${ }^{3, *}$ \\ 1 U.S. Army Research Laboratory, RDRL-VTM, Aberdeen Proving Ground, MD 21005-5069, USA; \\ jeffrey.l.gair.ctr@mail.mil (J.L.G.J.); daniel.p.cole.civ@mail.mil (D.P.C.); asha.j.hall.civ@mail.mil (A.J.H.); \\ mark.l.bundy2.civ@mail.mil (M.L.B.) \\ 2 Department of Mechanical Engineering, University of Maryland, College Park, MD 20742, USA; \\ bruck@umd.edu \\ 3 Department of Aeronautics and Astronautics, Massachusetts Institute of Technology, Cambridge, \\ MA 02139, USA; dlidston@mit.edu \\ 4 U.S. Army Research Laboratory, RDRL-WMM, Aberdeen Proving Ground, MD 21005-5069, USA; \\ robert.h.lambeth2.civ@mail.mil (R.H.L.); alex.j.hsieh.civ@mail.mil (A.J.H.) \\ * Correspondence: wardle@mit.edu; Tel.: +1-617-252-1539 \\ † Presented at the 18th Interaction Conference on Experimental Mechanics, Brussels, Belgium, 1-5 July 2018. \\ Published: 15 May 2018
}

\begin{abstract}
Poly(urethane-urea) (PUU) has been infused into ultrahigh volume fraction carbon nanotube (CNT) forests using a heat-curable polymer formula. Polymer nanocomposites with carbon nanotube volume-fractions of $1 \%, 5 \%, 10 \%, 20 \%$, and $30 \%$ were fabricated by overcoming densification and infusion obstacles. These polymer nanocomposites were nanoindented quasistatically and dynamically to discern process-structure-(mechanical) property relations of polymerizing PUU in such densely-packed CNT forests. A 100× increase in indentation modulus has been observed, which is attributed not only to CNT reinforcement of the matrix, but also to molecular interactions in the matrix itself. Quasi-static elastic moduli ranging from $10 \mathrm{MPa}-4.5$ GPa have been recorded. Storage modulus for all materials is found to track well at loadings of $200 \mathrm{~Hz}$, with little effect observed from increasing CNT volume fraction.
\end{abstract}

Keywords: carbon nanotubes; polymer nanocomposites; polyurethane urea; self-assembly

\section{Introduction}

Hierarchical composites comprising components of multiple length-scales are a promising area of research for the aerospace industry as they offer unparalleled control over material architecture. One method for fabricating such materials is to grow a conformal coating of carbon nanotubes (CNTs) off of the surfaces of advanced woven fabrics such as carbon fiber, aluminum oxide, etc. $[1,2]$. An additional level of control can be offered by engineering the interface between the chosen matrix material and the CNTs. This interphase region has been shown to be affected by CNT proximity [3-9]. Furthermore, packing and confinement effects which arise from even moderate CNT volume fractions $\left(\mathrm{V}_{\mathrm{f}}^{\prime} \mathrm{s}\right)$ give rise to additional nanostructure [10]. To investigate the phenomena governing this interphase, a model system of vertically aligned CNT forests has been 
used, which allows for excellent control of CNT $V_{f}$ and dispersion, while eliminating any potentially confounding effects of more complex hierarchical composites.

The matrix material used here is a poly(urethane-urea) (PUU) which has been investigated previously [11-16], and is particularly attractive for its quasi-static and dynamic mechanical tunability. Moderate interactions have been observed previously with this material in the presence of aligned CNTs $[17,18]$, and so a deeper dive into understanding the nature of this interaction and the mechanical implications is necessary. Quasi-static and dynamic nanoindentation results will be presented here.

\section{Methods}

CNT forests were grown at $1 \%$ volume fraction on silicon wafers using atmospheric pressure chemical vapor deposition. These CNT forests were biaxially densified down to the requisite dimensions for producing the desired CNT $V_{\text {f. }}$ The densified forests were placed into silicone molds to confine the samples and prevent expansion during polymer infusion. Poly(urethane-urea) (PUU) prepolymer was synthesized using a blocked-isocyanate, diamine, and polyol identical to the method outlined previously [18]. The mixed and degassed prepolymer was poured onto the densified CNT forests and heated at atmospheric pressure to $130{ }^{\circ} \mathrm{C}$ for $24 \mathrm{~h}$ per the previously prescribed cure cycle [18]. Two stoichiometric variations of PUU were used in the present study: PUU211 and PUU541, where the naming denotes HDMI:DETA:PTMO ratios.

PUU nanocomposites were made with 1\%, 5\%, 10\%, 20\%, and 30\% CNT V. Once samples were cured, they were removed from the molds and ultra-cryotomed to reveal smooth surfaces for the purpose of nanoindentation and atomic force microscopy. Sample were prepared so that both the longitudinal (L) and transverse (T) CNT orientations could be tested independently in hopes of elucidating the anisotropy inherent to these ordered structures.

Samples were imaged using a Cypher Scanning Probe Microscope. The silicon tip had a natural frequency of $70 \mathrm{kHz}$ and stiffness of $2 \mathrm{~N} / \mathrm{m}$, and was used to perform alternating contact mode scans at a rate of $0.6 \mathrm{~Hz}$.

Nanoindentation tests were performed using a Hysitron TI950 Triboindenter with a Berkovich tip. Quasi-static load functions with a $5 \mathrm{~s}$ ramp to $200 \mu \mathrm{N}, 5 \mathrm{~s}$ hold, and $5 \mathrm{~s}$ ramp down were implemented. A $2 \times 2$ array of quasi-static indentations was tested resulting in 4 tests per sample. Dynamic load functions with similar times and loads were used, with the addition of a dynamic amplitude of $3 \mu \mathrm{N}$ at frequencies ranging from 1-200 Hz. A $1 \times 3$ array of dynamic indentations was tested resulting in 3 tests per sample.

\section{Results \& Discussion}

The polymer nanocomposites were found to be highly anisotropic. Indentation modulus and storage modulus data for PUU211 as a function of CNT V $\mathrm{f}$ is found in Figure 1. Similar plots for the PUU541 materials can be found in Figure 2. The longitudinally-oriented CNTs increased the indentation modulus from $60 \mathrm{MPa}$ in the neat PUU211 to up to $1900 \mathrm{MPa}$ in the $30 \% \mathrm{CNT} \mathrm{Vf}$ PUU211 PNC. The same material with transversely-oriented CNTs exhibited a modulus of only 700 MPa. The neat PUU541 was shown to have a modulus of $~ 1300 \mathrm{MPa}$, while PUU541 with $30 \%$ CNT $\mathrm{V}_{\mathrm{f}}$ had a modulus of $\sim 4300 \mathrm{MPa}$ and $2200 \mathrm{MPa}$ in the longitudinal and transverse orientations respectively. All materials were found to behave rate-independently, though with dynamic storage moduli following a similar trend to indentation moduli. 


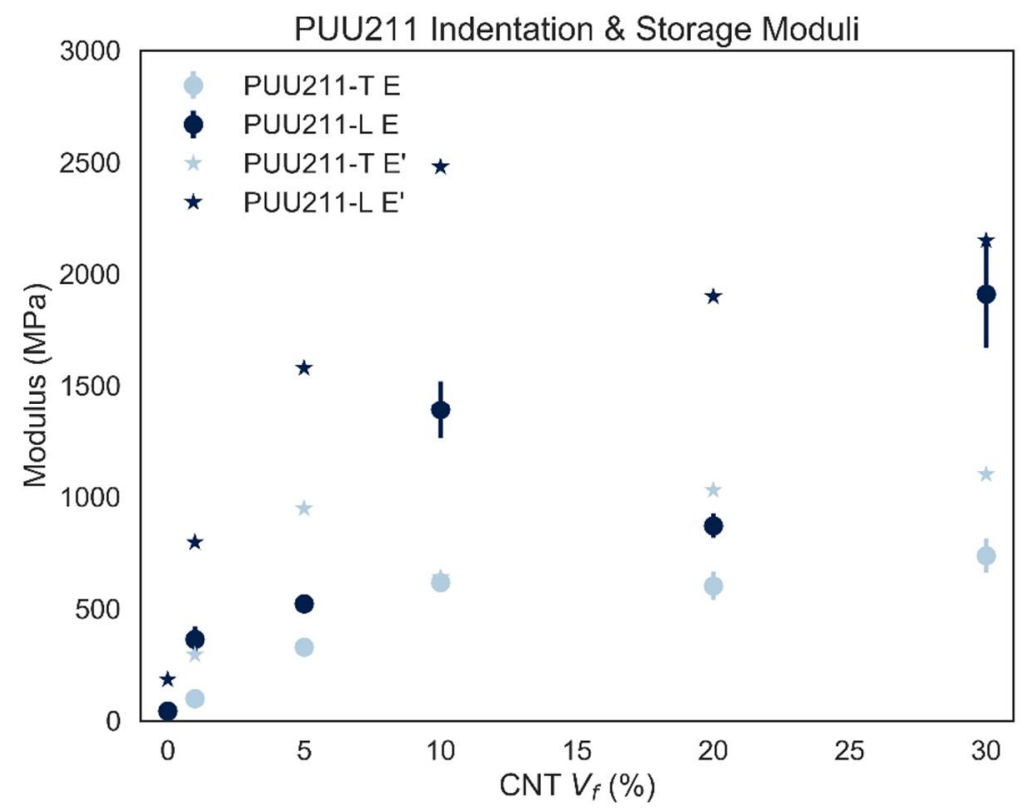

Figure 1. Comparison of quasi-static indentation modulus with average storage modulus (at $1 \mathrm{~Hz}$ ) for each PUU211 material. "T" represents "transverse" tests and " $\mathrm{L}$ " represents "longitudinal" tests. " $\mathrm{E}^{\prime \prime}$ is used to designate "indentation modulus," while " $\mathrm{E}^{\prime \prime}$ is used to designate "storage modulus".

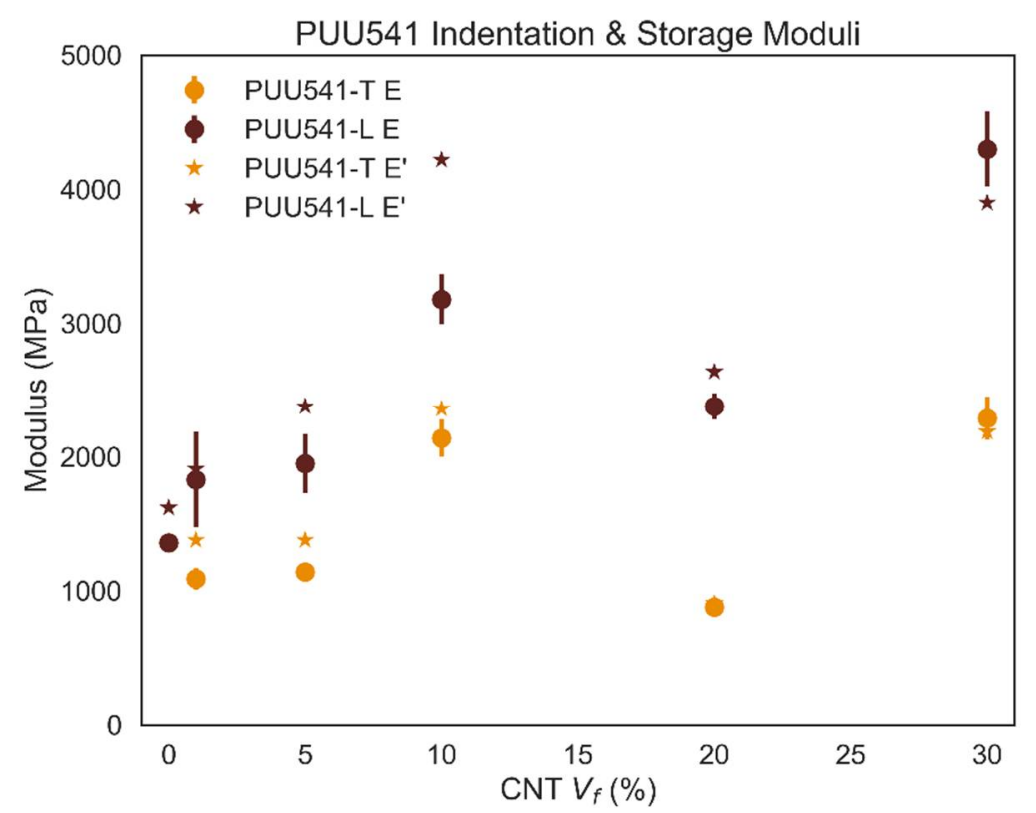

Figure 2. Comparison of quasi-static indentation modulus with average storage modulus (at $1 \mathrm{~Hz}$ ) for each PUU541 material. " $\mathrm{T}$ " represents "transverse" tests and " $\mathrm{L}$ " represents "longitudinal" tests. "E" is used to designate "indentation modulus," while " $\mathrm{E}^{\prime \prime}$ is used to designate "storage modulus".

In the case of each polymer, the $10 \% \mathrm{~V}_{\mathrm{f}}$ displayed a local maximum, while the $20 \% \mathrm{~V}_{\mathrm{f}} \mathrm{CNTs}$ exhibited a reduction in modulus. The AFM images of 10\% CNT V PUU541 PNC showed a highlyordered arrangement of nanophases surrounding the CNTs, while 20\% CNT Vf PUU541 PNC was much more amorphous. Examples of these may be found in Figure 3. The ordering found in the $10 \%$ CNT V $\mathrm{f}$ PUU541 PNC is thought to be a result of some optimized packing density at this CNT $V_{f}$. This appears to be a point which balances the mobility afforded by low CNT $V_{f}$ and the PUU hard segment ordering initiated by higher density CNT forests. At and above $20 \% \mathrm{~V}_{\mathrm{f}} \mathrm{CNTs}$, the PUU is unable to order itself due to the restriction of such densely-packed CNTs. Though poor 
ordering and hydrogen-bonding should proliferate at the $30 \% \mathrm{CNT} \mathrm{V}_{\mathrm{f}}$, the CNTs are so densely packed that their mechanical properties dominate material behavior in these materials.

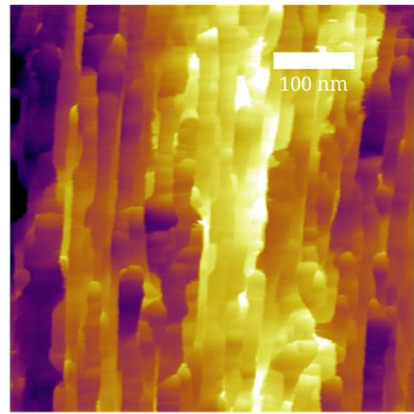

a

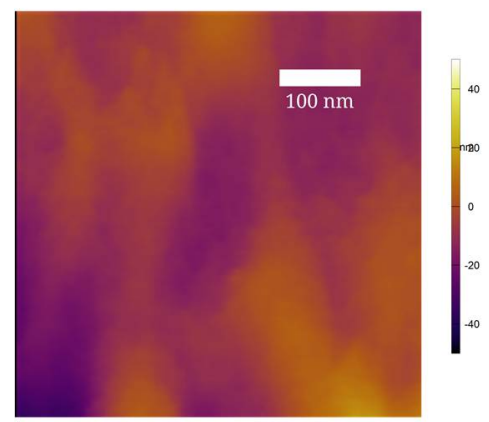

C

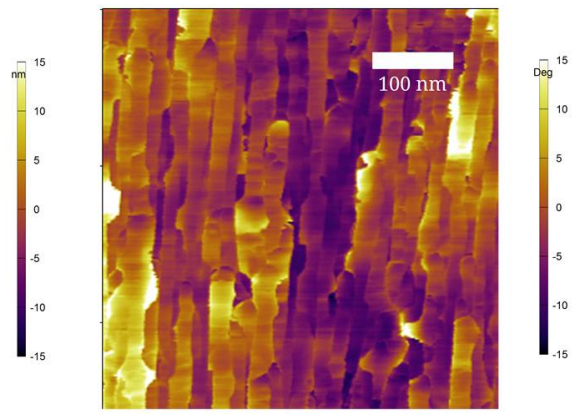

b

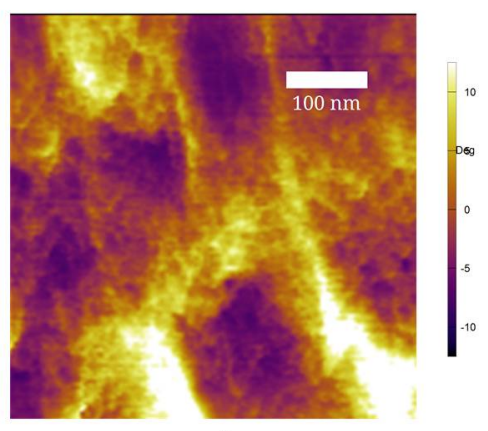

d

Figure 3. Characteristic AFM: (a) height and (b) phase maps for transversely-oriented PUU541 with $10 \%$ CNTs. (c) height and (d) phase maps for longitudinally-oriented PUU541 with $20 \%$ CNTs.

\section{Conclusions}

The CNTs were found to have a profound impact on the microscale mechanical properties of the PUU polymer nanocomposites, with significant anisotropy. An unexpected trend was observed with increasing $\mathrm{CNT} \mathrm{V}_{\mathrm{f}}$, which is expected to arise from different polymerization mechanisms governing each regime, giving rise to varied mechanical properties. Mechanical properties below $10 \% \mathrm{~V}_{\mathrm{f}}$ are found to be similar to one another, suggesting that the mechanical properties of these materials is largely matrix-dominated. Around $10 \% \mathrm{~V}_{\mathrm{f}}$ the materials stiffen significantly, which coincides with packed nanophases seen in AFM. This indicates that some ideal packing has occurred for the ordered and oriented hard-segments. Poor polymer chain mobility is thought to cause the reduced stiffness at $20 \%$, and CNT-packing is thought to dominate at $30 \% \mathrm{~V}_{\mathrm{f}}$.

Author Contributions: J.L.G.J., D.L.L., and B.L.W. conceived and designed the experiments. J.L.G.J., D.L.L., and B.L.W. conceived of and performed fabrication methods. J.L.G.J., D.L.L., and D.P.C. performed nanoindentation experiments. D.P.C. performed AFM experiments. All authors contributed to interpreting data and relating findings to a broader body of work. J.L.G.J. wrote the paper with input from B.L.W.

Acknowledgments: This project was supported in part by an appointment to the Research Participation Program at the U.S. Army Research Laboratory administered by the Oak Ridge Institute for Science and Education through an interagency agreement between the U.S. Department of Defense and the EPA. This work was also supported by Airbus, Embraer, Lockheed Martin, Saab AB, Hexcel, Saertex, TohoTenax, and ANSYS through MIT's Nano-Engineered Composite Aerospace STructures (NECST) Consortium, and was supported in part by the U.S. Army Research Laboratory and the U. S. Army Research Office through the Institute of Soldier Nanotechnologies, under contract number W911NF-13-D-0001. 


\section{References}

1. Yamamoto, N.; Hart, A.J.; Garcia, E.J.; Wicks, S.S.; Duong, H.M.; Slocum, A.H.; Wardle, B.L. High-yield growth and morphology control of aligned carbon nanotubes on ceramic fibers for multifunctional enhancement of structural composites. Carbon N. Y. 2009, 47, 551-560.

2. Li, R.; Lachman, N.; Florin, P.; Wagner, H.D.; Wardle, B.L. Hierarchical carbon nanotube carbon fiber unidirectional composites with preserved tensile and interfacial properties. Compos. Sci. Technol. 2015, 117, 139-145.

3. Wang, T.L.; Yu, C.C.; Yang, C.H.; Shieh, Y.T.; Tsai, Y.Z.; Wang, N.F. Preparation, characterization, and properties of polyurethane-grafted multiwalled carbon nanotubes and derived polyurethane nanocomposites. J. Nanomater. 2011, 2011, 7.

4. Xu, M.; Zhang, T.; Gu, B.; Wu, J.; Chen, Q. Synthesis and Properties of Novel PolyurethaneUrea/Multiwalled Carbon Nanotube Composites. Macromolecules 2006, 39, 3540-3545.

5. Chen, Y.; Zhang, B.; Gao, Z.; Chen, C.; Zhao, S.; Qin, Y. Functionalization of multiwalled carbon nanotubes with uniform polyurea coatings by molecular layer deposition. Carbon N. Y. 2015, 82, 470-478.

6. Pedrazzoli, D.; Manas-Zloczower, I. Understanding phase separation and morphology in thermoplastic polyurethanes nanocomposites. Polymer 2016, 90, 256-263.

7. Wu, H.L.; Wang, C.H.; Ma, C.C.M.; Chiu, Y.C.; Chiang, M.T.; Chiang, C.L. Preparations and properties of maleic acid and maleic anhydride functionalized multiwall carbon nanotube/poly(urea urethane) nanocomposites. Compos. Sci. Technol. 2007, 67, 1854-1860.

8. Patton, S.T.; Frasca, A.J.; Hu, J.; Varshney, V.; Phillips, B.S.; Roy, A.K.; Voevodin, A.A. Multiphysics characterization of multi-walled carbon nanotube thermoplastic polyurethane polymer nanocomposites during compression. Carbon N. Y. 2016, 98, 638-648.

9. Fernández-d'Arlas, B.; Khan, U.; Rueda, L.; Coleman, J.N.; Mondragon, I.; Corcuera, M.A.; Eceiza, A. Influence of hard segment content and nature on polyurethane/multiwalled carbon nanotube composites. Compos. Sci. Technol. 2011, 71, 1030-1038.

10. Wardle, B.L.; Saito, D.S.; García, E.J.; Hart, A.J.; De Villoria, R.G.; Verploegen, E.A. Fabrication and characterization of ultrahigh-volume-fraction aligned carbon nanotube-polymer composites. Adv. Mater. 2008, 20, 2707-2714.

11. Sarva, S.S.; Deschanel, S.; Boyce, M.C.; Chen, W. Stress-strain behavior of a polyurea and a polyurethane from low to high strain rates. Polymer 2007, 48, 2208-2213.

12. Chantawansri, T.L.; Sliozberg, Y.R.; Andzelm, J.W.; Hsieh, A.J. Coarse-grained modeling of model poly(urethane urea)s: Microstructure and interface aspects. Polymer 2012, 53, 4512-4524.

13. Strawhecker, K.E.; Hsieh, A.J.; Chantawansri, T.L.; Kalcioglu, Z.I.; Van Vliet, K.J. Influence of microstructure on micro-/nano-mechanical measurements of select model transparent poly(urethane urea) elastomers. Polymer 2013, 54, 901-908.

14. Hsieh, A.J.; Orlicki, J.A.; Beyer, R.L. Molecular Design of Novel Poly (Urethane-Urea) Hybrids as Helmet Pads for Ballistic and Blast Trauma Mitigation; ARL Technical Reports; Army Research Laboratory: Aberdeen Proving Ground, MD, USA, 2105.

15. Primeaux, D.J. Polyurea vs. Polyurethane \& Polyurethane/Polyurea: What's the Difference? In Proceedings of the Polyurea Linings Annual Conference, Polyurea Development Assocaition (PDA), Tampa, FL, USA, 2-4 March 2004.

16. Kantheti, S.; Gaddam, R.R.; Narayan, R.; Raju, K.V.S.N. Hyperbranched polyol decorated carbon nanotube by click chemistry for functional polyurethane urea hybrid composites. RSC Adv. 2014, 4, 24420-24427.

17. Gair, J.L.; Lambeth, R.H.; Cole, D.P.; Lidston, D.L.; Hsieh, A.J.; Bruck, H.A.; Bundy; Wardle, B.L. Tunable Poly(Urethane-Urea) Based Aligned Carbon-Nanotube Polymer Nanocomposites. In Proceedings of the 21 st International Conference on Composite Materials, Xi'an, China, 20-25 August 2017.

18. Gair, J.L.; Lambeth, R.H.; Cole, D.P.; Lidston, D.L.; Stein, I.Y.; Kalfon-Cohen, E.; Hsieh, A.J.; Bruck, H.A.; Bundy, M.L.; Wardle, B.L. Strong Process-Structure Interaction in Stoveable Pol(Urethane-Urea) Aligned Carbon Nanotube Nanocomposites. Compos. Sci. Technol. 2018, submitted.

(C) 2018 by the authors. Licensee MDPI, Basel, Switzerland. This article is an open access article distributed under the terms and conditions of the Creative Commons Attribution (CC BY) license (http://creativecommons.org/licenses/by/4.0/). 